\title{
INSTABILITÉ DE BÉNARD-MARANGONI DANS LES NÉMATIQUES
}

\author{
E. GUYON $(*)$
}

\author{
Laboratoire de Physique des Solides, Université Paris-Sud, 91405 Orsay, France
}

\section{G. VELARDE (*)}

\begin{abstract}
Departament de Fisica, Universidad Autonoma de Madrid, Cantoblanco (Madrid) Espagne
\end{abstract}
(Reçu le 7 avril 1978, accepté le 17 mai 1978)

\begin{abstract}
Résumé. - Nous discutons théoriquement l'effet déstabilisant de la tension superficielle (instabilité Bénard-Marangoni) et de la poussée d'Archimède (Rayleigh-Bénard) sur une couche mince, horizontale, libre, chauffée par le bas, de nématique en géométrie planaire. Le mécanisme d'instabilité est décrit à partir d'un modèle simplifié en bon accord avec les résultats d'un calcul numérique.
\end{abstract}

\footnotetext{
Abstract. - We discuss theoretically the destabilizing effects of surface tension traction (BénardMarangoni) and buoyancy (Rayleigh-Bénard) on a planar nematic free thin layer heated from below. The mechanism of instability is described using a simplified model. It agrees with results of a more detailed numerical calculation.
}

On considère une couche mince horizontale (limitée par les plans $z=0, d$ ) de cristal liquide nématique planaire (la direction moyenne des molécules parallèles à $x$ est définie par l'ancrage aux surfaces limites)' chauffée par en dessous ; $\beta=[T(z=0)-T(z=d)] / d$ est positif (Fig. 1). Au-dessus d'un gradient critique $\beta_{\mathrm{c}}$ apparaît une distorsion du directeur, périodique dans la direction $x$, associée à un système de rouleaux convectifs d'axes perpendiculaires à $x$ et de même période. Par rapport aux instabilités classiques de Rayleigh-Bénard des liquides isotropes [1], l'originalité du cas nématique [2] vient de ce que la distorsion (caractérisée par la composante verticale du directeur, $n_{z}, n_{z} \ll|\mathbf{n}|$ ) produit une distribution inhomogène de température $T(x)$ due à l'anisotropie du tenseur de conductivité thermique (l'anisotropie de conductivité thermique $k_{\mathrm{a}}=k_{\|}-k_{\perp}$ est positive; $/ /$ et $\perp$ se rapportent à l'orientation du flux de chaleur par rapport à n) ; sous l'action de la poussée d'Archimède une vitesse locale est induite dont le gradient tend à renforcer la fluctuation. initiale du. directeur. Jusqu'à présent, on a considéré seulement l'effet déstabilisant de la poussée d'Archimède (le coefficient de dilatation $\alpha=\frac{1}{v}\left(\frac{\partial v}{\partial T}\right)_{p}$ est positif). Dans cette note, nous discutons l'effet additionnel de la tension superficielle - ou effet Marangoni - présent lorsque la

(*) Laboratoire de Dynamique et Thermodynamique des Fluides, Université de Provence, Marseille, France.

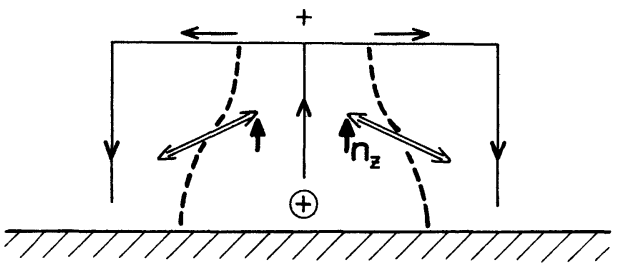

FIG: 1. - Géométrie et mécanisme de l'instabilité de RayleighBénard dáns les nématiques. Partant d'une fluctuation de températuree (' + ') en surface, un mouvement de traction en surface est induit sous l'effet de la variation de tension superficielle (le signe des vitesses correspond au cas normal où la tension superficielle décroît lorsque $T$ croît). Ceci induit un mouvement convectif en volume. L'apport convectif de chaleur terme $\beta w$ dans (2) renforce la fluctuation dans le cas isotrope. Dans le cas nématique, le mécanisme dominant vient de la distorsion, caractérisée par la composante (petite) $n_{z}$ du directeur, induite par les gradients de vitesse verticale. A cause du transfert de chaleur plus efficace le long de la direction moléculaire moyenne, la chaleur est focalisée (tirets) dans la région de courbure négative du directeur. Au mécanisme moteur en surface s'ajoute l'effet volumique de la poussée d'Archimède qui tend à faire monter le liquide moins dense (point chaud $\oplus$ ) dans les régions de plus en plus froides.

[Geometry and mechanism of the Rayleigh-Benard instability in nematics. Starting from a temperature fluctuation $(+)$ at the free surface, a surface traction is induced due to the gradient of surface tension $\sigma$ (the sign of velocities corresponds to the usual case where $\sigma$ is a decreasing function of $T$ ). A convective motion $(\beta w$ in (2)) is induced in the bulk and reinforces the initial fluctuation in the isotropic case. In nematics, the dominant mechanism comes from the distortion, characterized by the (small) component $n_{z}$ of the director. Thanks to the more efficient transfer of heat along $\mathbf{n}$, heat is focused (broken lines) in the region of negative curvature of the director. A buoyancy effect which tends to push the warmer liquid $\oplus$ towards the colder upper regions should be added to the description.] 
face supérieure du nématique est libre (le coefficient $\sigma^{\prime}=-(\mathrm{d} \sigma / \mathrm{d} T)$, où $\sigma$ est la tension superficielle, est positif). Nous renvoyons à la référence [3] pour une première discussion de l'effet Marangoni dans un nématique. Cependant, à la différence de cette étude :

i) nous considérons l'effet premier d'un gradient de température vertical (qui, en régime convectif, donnera lieu à des gradients horizontaux secondaires $T(x))$ et non celui d'un gradient horizontal qui était obtenu, dans la référence [3], par l'impact d'un faisceau laser sur une goutte nématique.

ii) Nous supposons que l'orientation du directeur à la surface libre est planaire, ce qui est obtenu dans certains nématiques. Dans le cas du matériau de la référence [3], il existait un angle fini de raccordement qui joue un rôle crucial dans la description des effets observés. Un tel ancrage conique est susceptible de modifier les résultats de l'analyse que nous décrivons par l'existence de couples hydrodynamiques d'axe vertical.

Nous discutons dans cette note un calcul simplifié qui permet de dégager les mécanismes de l'instabilité et donne un ordre de grandeur des seuils en bon accord avec un calcul détaillé à paraître dont nous indiquons les résultats. En régime convectif, on ne retient que la dépendance perpendiculaire à la direction des rouleaux des fluctuations de la composante verticale de la vitesse, de la température et du directeur

$$
w / w_{0}=\theta / \theta_{0}=n_{z} / n_{z_{0}}=\mathrm{e}^{i q x}
$$

et, en accord qualitatif avec l'expérience, on ne considère, au voisinage immédiat du seuil

$$
\left(|w|,|\theta|,\left|n_{z}\right| \ll 1\right),
$$

qu'un seul mode instable $q=\pi / d$ (la taille des rouleaux est de l'ordre de grandeur de l'épaisseur).

Une telle méthode simplifiée unidimensionnelle a été utilisée avec succès dans la description de seuils de nombreux problèmes [ $2 a, 4]$.

Pour les équations de la chaleur et du directeur nous reprenons les expressions données par E. DuboisViolette $[2 a, c]$. (L'effet de la poussée d'Archimède, dans l'équation des forces, qui complète la description du problème Rayleigh-Bénard, sera considéré plus loin (Eq. (6))) :

$$
\begin{array}{r}
\frac{\partial \theta}{\partial t}-\beta w-\tilde{\kappa} \frac{\partial^{2} \theta}{\partial x^{2}}+\kappa_{\mathrm{a}} \beta \frac{\partial n_{z}}{\partial x}=0 \\
\gamma \frac{\partial n_{z}}{\partial t}+\alpha_{2} \frac{\partial w}{\partial x}-\tilde{K} \frac{\partial^{2} n_{z}}{\partial x^{2}}=0
\end{array}
$$

$\tilde{\kappa}=k / \rho C$ est la diffusivité thermique; $\gamma$ est une viscosité de rotation du directeur; $\alpha_{2}$ est le coefficient de viscosité associé au couple visqueux tendant à faire tourner, dans le sens donné par la figure 1, le directeur sous l'effet du cisaillement $\partial w / \partial x$ (on peut négliger le couple beaucoup plus petit en général dû au gradient de vitesse $\partial u / \partial z$ où $u$ est la composante horizontale de la vitesse); $\tilde{K}$ est une constante élastique moyenne limitant la distorsion du directeur. Le signe indique des moyennes sur des constantes qui pourraient être évaluées simplement dans des modèles bidimensionnels. Le terme $\kappa_{\mathrm{a}} \beta \frac{\partial n_{z}}{\partial x}$ décrit le couplage entre la courbure du directeur et la fluctuation de température : focalisation thermique.

Nous négligeons provisoirement la poussée d'Archimède (en faisant $g=0$ ). Le seul mécanisme déstabilisant est la variation de la tension superficielle dont l'effet est décrit qualitativement dans la légende de la figure 1. Dans le cadre du modèle unidimensionnel (ce qui, a priori, est une extrapolation hardie alors qu'il s'agit d'une condition aux limites !) nous écrivons l'équilibre des tensions hydrodynamiques en surface :

$$
\sigma^{\prime}\left(\frac{\partial \theta}{\partial x}\right)_{z=d}=-\tilde{\eta}\left(\frac{\partial u}{\partial z}\right)_{z=d} .
$$

On peut ramener l'expression du gradient de la composante de vitesse $u$ à celui de $w$ en exprimant l'équation de continuité

$$
\frac{\partial u}{\partial x}+\frac{\partial w}{\partial z}=0
$$

et en faisant une hypothèse de rouleaux carrés qui permet d'écrire en ordre de grandeur

$$
\frac{\partial u}{\partial z} \sim-\frac{\partial w}{\partial x} \quad \text { (hypothèse A) }
$$

d'où

$$
\sigma^{\prime}\left(\frac{\partial \theta}{\partial x}\right)_{z=d} \sim \tilde{\eta}\left(\frac{\partial w}{\partial x}\right) .
$$

En utilisant l'analyse en modes normaux (Eq. (1)) et en supposant (principe d'échange des stabilités) que, au voisinage d'un seuil linéaire, $\partial / \partial t=0$, on obtient le système de 3 équations homogènes

$$
\begin{aligned}
\tilde{\kappa} q^{2} \theta_{0}-\beta w_{0}+\kappa_{\mathrm{a}} \beta i q n_{z_{0}} & =0 \\
\alpha_{2} i q w_{0}+\tilde{K} q^{2} n_{z_{0}} & =0 \\
+\sigma^{\prime} i q \theta_{0}-\tilde{\eta} i q w_{0} & =0 .
\end{aligned}
$$

La condition de compatibilité nous donne le seuil en présence de phénomènes interfaciaux

$$
\beta_{\mathrm{c}}=\frac{\tilde{\kappa} \tilde{\eta} q^{2}}{\sigma^{\prime}\left(1-\kappa_{\mathrm{a}} \alpha_{2} / \tilde{K}\right)}
$$

On retrouve la définition du nombre de Marangoni, $M=\beta \sigma^{\prime} d^{2} / \tilde{\kappa} \tilde{\eta}$, qui caractérise le seuil de convection pour un liquide isotrope. Le facteur anisotrope $1-\kappa_{\mathrm{a}} \alpha_{2} / \tilde{K}$, qui est aussi obtenu dans le traitement des effets de pesanteur [2], est très supérieur à 1 (typiquement de l'ordre de 300 ; notons que $\alpha_{2}$ est 
négatif [5]). Cette diminution très importante du seuil convectif est due au fait que le couplage avec la distorsion du directeur introduit, dans le mécanisme convectif, la constante de temps de relaxation du directeur $\left(\tilde{K} q^{2} / \alpha_{2}\right)^{-1}$ très longue devant la constante de temps thermique qui donne la relaxation dans le cas isotrope (mesuré par $\left.\left(\tilde{\kappa} q^{2}\right)^{-1} \sim\left(\kappa_{\mathrm{a}} q^{2}\right)^{-1}\right)$.

Il est possible de rajouter l'effet de la pesanteur dans le cadre de ce traitement simplifié. Dans le cas où $\sigma^{\prime}=0$ et $g \neq 0$, l'équation de N.S. appliquée au nématique donne :

$$
\rho \frac{\partial w}{\partial t}=+\alpha \rho g \theta+\tilde{\eta}^{\prime} \frac{\partial^{2} w}{\partial x^{2}}+\alpha_{2} \frac{\partial}{\partial t}\left(\frac{\partial n_{z}}{\partial x}\right)
$$

( a priori $\tilde{\eta}^{\prime} \neq \tilde{\eta}$ ) qui s'exprime au seuil par

$$
\alpha g \theta_{0}-\tilde{\eta}^{\prime} q^{2} w_{0}=0 .
$$

On peut superposer l'effet déstabilisant (hypothèse B) de la force de pesanteur $(\alpha \rho g \theta)$ et des tractions superficielles $\left(q \sigma^{\prime} \frac{\partial \theta}{\partial x}\right)$.

L'équation (4') est alors remplacée par :

$$
w_{0}-\left(\frac{\alpha g}{\tilde{\eta}^{\prime} q^{2}}+\frac{\sigma^{\prime}}{\tilde{\eta}^{*}}\right) \theta_{0}=0
$$

et l'expression générale du seuil (obtenue à partir de $\left.\left(2^{\prime}\right),\left(3^{\prime}\right),(7)\right)$ se met sous la forme particulièrement simple, valable dans les liquides isotropes $[6,7]$

$$
\frac{M}{M_{\mathrm{c}}}+\frac{R}{R_{\mathrm{c}}}=1
$$

où le nombre de Rayleigh

$$
R=\frac{\alpha \rho g \beta d^{4}}{\widetilde{\kappa} \widetilde{\eta}^{\prime}}
$$

et où les valeurs $M_{\mathrm{c}}$ et $R_{\mathrm{c}}$ pour les nématiques sont réduites par le facteur $\left(1-\frac{\kappa_{\mathrm{a}} \alpha_{2}}{\tilde{K}}\right)$ par rapport au cas isotrope.

La description très simplifiée donnée ci-dessus avait pour objet de dégager les mécanismes moteurs des instabilités convectives et les ordres de grandeur de seuil tout en s'appuyant sur des hypothèses (en particulier A et B) dont la justification repose sur l'analyse numérique faite indépendamment dont les calculs seront discutés dans une publication ultérieure [8].

En utilisant la méthode de Galerkin avec des fonctions d'essai comme celles décrites par Finlayson [9], nous avons obtenu, en première approximation, les résultats suivants :

a) $g=0$ (pas de gravitation) avec un coefficient $\sigma^{\prime} \sim 10^{-2} \mathrm{cgs}[10]$

$$
M_{\mathrm{c}}=\frac{\beta_{\mathrm{c}} d^{2} \sigma^{\prime}}{\tilde{\kappa} \tilde{\eta}}=1,9 \quad \text { et } \quad a_{\mathrm{c}}=q_{\mathrm{c}} d=1,5
$$

Cette valeur se compare à la valeur $M_{\mathrm{c}}=80\left(a_{\mathrm{c}}=1,99\right)$ pour un liquide isotrope de mêmes propriétés moyennes.

b) $\sigma^{\prime}=0$ (ce cas est obtenu en particulier en l'absence de phénomènes interfaciaux)

$$
R_{\mathrm{c}}=\frac{\alpha \rho g \beta_{\mathrm{c}} d^{4}}{\widetilde{\kappa} \tilde{\eta}^{\prime}} \sim 26 \quad\left(a_{\mathrm{c}}=3,01\right) .
$$

Le nombre critique est en très bon accord avec ceux obtenus par Dubois-Violette [2c] et Barratt et Sloan [11] et est aussi très inférieur au seuil isotrope qui est $R_{\mathrm{c}}=669\left(a_{\mathrm{c}}=1,1\right)$ si la paroi supérieure est libre et mauvaise conductrice ou $R_{\mathrm{c}}=1100\left(a_{\mathrm{c}}=2,7\right) \mathrm{si}$ elle est libre et bonne conductrice.

Notons que le rapport des nombres $R_{\mathrm{c}}$ en phase isotrope et nématique est le même que celui des nombres $M_{\mathrm{c}}$, en accord avec le modèle simplifié.

c) L'analyse, dans le cas général, redonne la formule (8) obtenue avec le modèle simplifié précédent. Dans le cas d'un film mince, il y aura prédominance de l'effet Marangoni (à cause de la dépendance en $d^{2}$ au lieu de $d^{4}$ ). Typiquement, pour un film nématique de $1 \mathrm{~mm}$ d'épaisseur, le mécanisme de Marangoni donne un seuil $\beta_{\mathrm{c}} \sim 1,9 \mathrm{degré} / \mathrm{cm}$.

Le calcul et les expériences [2] en l'absence d'effet de tension superficielle donnent, pour la même épaisseur, une valeur de seuil plus élevée $\beta_{\mathrm{c}} \sim 30$ degrés $/ \mathrm{cm}$. L'effet dominant est donc, dans ce cas et en présence d'une surface libre, celui des tractions de surface.

La faible valeur du seuil de Marangoni pour les nématiques et le couplage avec la distorsion qui permet de visualiser de façon très sensible et directe une carte de vitesse et de température peuvent donner lieu à des applications de visualisation, dans un esprit un peu comparable à une remarquable expérience de visualisation infrarouge [12] utilisant une couche libre de, liquide isotrope en présence d'un gradient horizontal de température imposé (l'image infrarouge). Dans cette expérience, l'existence d'une couche liquide supplémentaire est nécessaire pour révéler les inhomogénéités du champ de vitesse convectif. Compte tenu de la possibilité d'avoir des seuils convectifs pour des très faibles épaisseurs nématiques, on peut penser à l'utilisation de l'instabilité de Bénard nématique à la thermographie I.R. (la résolution de l'image étant de l'ordre de la taille d'un rouleau, soit $d$ ). Si on se place au voisinage immédiat du seuil, la fluctuation initiale créée par un champ thermique horizontal appliquée à la force supérieure du nématique serait alors amplifiée par le mécanisme convectif. Des expériences sont en cours.

Nous tenons à remercier M. J. C. Antoranz pour son aide avec le calcul numérique et la tabulation des intégrales de Galerkin. Nous avons bénéficié de discussions avec E. Dubois-Violette, J. Pantaloni et $\mathbf{P}$. Pieranski et avec les physiciens de l'Institut 
d'Optique d'Orsay travaillant sur les applications de l'effet Marangoni à la thermographie I.R. Les rapports critiques des deux rapporteurs nous ont été très précieux.

\section{Bibliographie}

[1] Normand, C., Pomeau, Y. et Velarde, M. G., Rev. Mod. Phys. 49 (1977) 581 ; contient une bibliographie exhaustite sur les mécanismes de gravité et de tension superficielle dans la convection thermique.

[2a] Dubois-Violette, E., C.R. Acad. Sci. 273 (1971) 923.

[2b] Guyon, E. et Pieranski, P., C. R. Acad. Sci. 274 (1972) 656.

[2c] Dubois-Violette, E., Solid State Commun. 14 (1974) 767,

[3] Urbach, W., Rondelez, F., Pieranski, P. et Rothen, F., J. Physique 38 (1977) 1275 ; on y trouvera une bibliographie sur les études de surface libre d'un nématique.

[4] Guyon, E. et Pieranski, P., Physica 73 (1974) 404.

[5] Les valeurs numériques estimées pour MBBA et dans le cadre des approximations sur $\sim$ valables pour un modèle unidimensionnel donnent (unités cgs)

$\tilde{K} \sim 7 \times 10^{-7} ; \tilde{\kappa}=\kappa_{\|} \sim 2 \kappa_{\mathrm{a}} \sim 10^{-3} ;$

$$
-\alpha_{2} \sim \gamma_{1} \sim 0.5: \tilde{\eta} \sim 0,1 \text {. }
$$

[6] Nield, D. A., J. Fluid. Mech. 19 (1964) 341.

[7] Pantaloni, J., Bailleux, R., Salan, J. et Velarde, M. G., soumis à $J$. Non Equilib. Thermodyn.

[8] ZuÑEGa, I. et Velarde, M. G., (à soumettre pour publication au $J$. Physique).

[9] Finlayson, B. A., The method of weighted residuals and variational principles (Acad. Press New York), 1972, chap. 6.

[10] Proust, J., Communication privée.

[11] Barratt, P. J. et Sloan, D. M., J. Phys. A 9 (1976) 1987.

[12] Lollergue, J. C.. Imbert, C., Levy, Y.. Communication présentée à l'Optical Society of America (10 octobre 77, Toronto). 